\title{
Aparelho Intraoral de Controle Lingual para o Controle da SAOS Grave
}

\author{
Oral Appliance Lingual for Severe OSA Control
}

\author{
Marilene de Oliveira Trindade ${ }^{a^{*}}$; Lucas Carvalho Aragão Albuquerque ${ }^{\mathrm{b}}$; Jorge Machado Caram ${ }^{\mathrm{c}}$
}

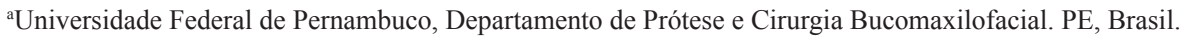

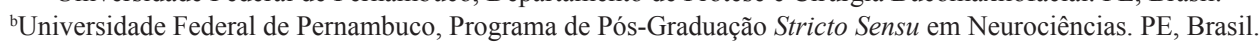 \\ ${ }^{\mathrm{c} A s s o c i a c ̧ a ̃ o ~ B r a s i l e i r a ~ d o ~ S o n o ~(A B S) . ~ B e l o ~ M G, ~ B r a s i l . ~}$ \\ "E-mail: marilene08@hotmail.com \\ Recebido em: 05/10/2015; Aceito em: 26/11/2015
}

\begin{abstract}
Resumo
A Síndrome da Apneia e Hipopneia Obstrutiva do Sono - SAOS é um distúrbio respiratório do sono caracterizado por episódios recorrentes de obstrução total ou parcial da via aérea superior durante o sono. Os sintomas mais comuns são: ronco, sonolência excessiva e prejuízos na concentração. A administração da pressão positiva contínua da via aérea CPAP é atualmente o padrão-ouro para o tratamento da SAOS. Contudo, os pacientes que o utilizam não têm assegurada sua adesão satisfatória ao tratamento. A praticidade e o relativo conforto fazem com que os Aparelhos Intraorais - AIO sejam mais aceitos pelos pacientes. O mais usado é o de avanço mandibular, mas possui algumas limitações na aplicação, como: pacientes com próteses extensas, poucos elementos dentários e problemas na articulação temporomandibular - ATM. Este trabalho tem como finalidade avaliar a aplicabilidade e a dinâmica mandibular após o uso do AIO em um paciente de 65 anos de idade com SAOS grave. Após a realização da polissonografia - PSG o paciente foi submetido ao uso do AIO de controle lingual. Após cinco meses de uso do AIO o paciente foi reavaliado com ajuda do mapeamento gráfico computadorizado e milimétrico da movimentação mandibular. O resultado revelou melhora significativa no quadro apnéico do paciente e poucas alterações na dinâmica mandibular e mastigatória. Os achados deste trabalho indicam que o AIO de controle lingual pode ser eficaz no tratamento da SAOS de grau severo. Além disso, o AIO não acarretou prejuízos às funções estomatognáticas nem movimentação mandibular. Mais estudos devem sem realizados para corroborar os resultados aqui demonstrados.
\end{abstract}

Palavras-chave: Transtornos do Sono. Apneia do Sono Tipo Obstrutiva. Qualidade de Vida.

\begin{abstract}
Obstructive sleep apnea-hypopnea syndrome (OSAHS) is a sleep-disordered breathing characterized by recurrent episodes of partial or complete obstruction of the upper airways. The most common symptoms are snoring, excessive sleepiness and impaired concentration. Continuous positive airway pressure (CPAP) is currently the gold standard for the treatment of OSA. However, this form of treatment does not ensure patient adherence and satisfaction. The convenience and relative comfort make intraoral appliances more acceptable to patients. $A$ mandibular advancement device is the most commonly employed intraoral appliance, but has limited effect for patients with extensive dentures, few teeth in the oral cavity and problems with the temporomandibular joint. The aim of the present study was to evaluate the applicability of an intraoral appliance and subsequent mandibular dynamics in a patient aged 65 years with severe OSA. The patient was submitted to polysomnography and the placement of a tong control device. After five months of usage, the patient was reevaluated using computerized and millimeter graphic mapping of mandibular movements. At the end of five months of treatment, a significant improvement occurred in OSA, with few alterations to mandibular and masticatory dynamics. The findings of the present study demonstrate that a tong control intraoral device constitutes in an effective treatment for severe OSA and does not causes significant impairment to stomatognathic function or mandibular movements. More studies should be made for corroborate the results shown here.
\end{abstract}

Keywords: Sleep Disorders. Sleep Apnea, Obstructive. Quality of Life.

\section{Introdução}

A Síndrome da Apneia Obstrutiva do Sono - SAOS é um distúrbio respiratório caracterizado por episódios recorrentes de obstrução total ou parcial das vias aéreas superior durante o sono, que leva à hipoxemia intermitente, hipercapnia transitória e despertares frequentes. $\mathrm{O}$ gênero, o índice da massa corpórea, os fatores genéticos, anatômicos, hormonais e o controle da ventilação interagem diversamente na fisiopatologia e expressão clínica da doença. A SAOS foi incluída como fator causal para a hipertensão arterial sistêmica, acidente vascular cerebral, arritmias e doença cardíaca coronariana, assim como mortalidade cardiovascular ${ }^{7}$.

A prevalência da SAOS, de moderada a grave, nos Estados Unidos nos períodos de 2007 a 2010 foi estimada em $10 \%$ em mulheres e $17 \%$ em homens entre 30 e 49 anos, representando um aumento substancial entre $14 \%$ e $55 \%$, dependendo do subgrupo estudado.

A administração da pressão positiva contínua da via aérea - CPAP constitui-se no padrão-ouro para o tratamento da SAOS. Os pacientes que a utilizam não têm assegurada sua adesão ao tratamento. A praticidade e o relativo conforto fazem com que os Aparelhos Intraorais - AIO sejam mais aceitos e indicados para ronco e SAOS de leve a moderada 
ou para pacientes com SAOS grave que não se adaptam ao CPAP.

O Aparelho de Controle Lingual é baseado no controle de posicionamento da língua, indicado para pacientes com quantidade de dentes insuficientes para ancoragem do dispositivo, pacientes edêntulos, com reabilitações protéticas extensas, problemas periodontais, presença de disfunção temporomandibular e/ou bruxismo. Um dos efeitos colaterais mais observados com o uso do AIO de um modo geral tem relação com alterações da posição mandibular, posições dentárias e complicações na forma de dor por DTM.

A eletrognatografia - EGN trata-se de um método para traçar os movimentos mandibulares, servindo como auxiliar no diagnóstico das DTM. O objetivo deste trabalho foi relatar a instalação e o acompanhamento do AIO de Controle Lingual em um paciente portador de SAOS grave, sendo utilizada a eletrognatografia antes e após seis meses de uso do aparelho para avaliação da dinâmica mandibular ou alteração na amplitude dos movimentos.

\section{Material e Métodos}

Segundo a Associação Americana de Distúrbios do Sono - AADS, a Síndrome da Apneia e Hipopneia Obstrutiva do Sono - SAOS é um distúrbio respiratório caracterizado por episódios recorrentes de obstrução total ou parcial das vias aéreas superiores - VAS durante o sono $^{1}$, que leva a hipoxemia intermitente, hipercapnia transitória e despertares frequentes, associados a sinais e/ou sintomas clínicos ${ }^{2}$. Essa é uma doença de causa multifatorial e ainda não totalmente esclarecida $^{3}$.

A classificação da gravidade da SAOS é baseada em critérios clínicos e polissonográficos conforme o Índice de Apneia e Hipopneia - IAH, que indica o número de interrupções na respiração por hora de sono. Pode ser classificada em leve - IAH de 5 a 15 -, moderada - IAH entre 16 a 30 e grave - IAH maior que 30 eventos $^{4}$.

De acordo com a Classificação Internacional de Distúrbio do Sono (2005) o diagnóstico da SAOS no adulto requer a presença dos critérios $\mathrm{A} \mathrm{B}+\mathrm{D}$ ou $\mathrm{C}+\mathrm{D}$, descritos abaixo:

1) No mínimo um dos critérios abaixo:

$\checkmark$ Episódios de Sono não intencionais durante a vigília, sonolência diurna excessiva - SDE, sono não reparador, fadiga ou insônia;

$\checkmark$ Acordar com pausas respiratórias, engasgos ou asfixia;

$\checkmark$ Companheiro(a) relata ronco alto e/ou pausas respiratórias durante o sono;

$\checkmark$ PSG apresentando:

$\checkmark$ Cinco ou mais eventos respiratórios detectáveis por hora de sono;

$\checkmark$ Evidências de esforço respiratório durante todo ou parte de cada evento.

2) Polissonografia apresentando: $\checkmark$ Quinze ou mais eventos respiratórios detectáveis por hora de sono;

$\checkmark$ Evidência de esforço respiratório durante todo ou parte de cada evento.

O distúrbio não pode ser mais bem explicado por outro distúrbio do sono, doenças médicas ou neurológicas, uso de medicação ou por uso de substâncias.

O gênero, o índice da massa corpórea - IMC, os fatores genéticos, anatômicos, hormonais e o controle da ventilação interagem diversamente na fisiopatologia e expressão clínica da doença ${ }^{3}$. A maior parte dos pacientes diagnosticados com apneia apresenta sobrepeso e quanto maior o IMC, mais grave a apneia. A literatura sugere que o excesso de peso levaria ao estreitamento da faringe, ou por deposição de gordura nas paredes da faringe, ou então nas estruturas parafaríngeas, como língua, palato mole e úvula ${ }^{4}$.O achado individual mais relevante do exame físico em pacientes com ronco/AOS é a medida da circunferência cervical. A associação mais relevante do exame físico inclui o índice de massa corpórea, circunferência cervical e avaliação do esqueleto crâniofacial, chamado de modelo morfométrico ${ }^{4}$. Outros sintomas comuns incluem cefaleia matinal, presença de ronco, sono não reparador, fadiga e alterações cognitivas. As alterações anatômicas sobre a via aérea superior mais descritas são: alterações nasais, tonsilas palatinas hiperplásicas, Índice de Mallampati Modificado classes III e IV (inadequada relação entre a base da língua e a orofaringe $)^{5}$.

Estudos recentes demonstraram que mesmo os distúrbios do sono considerados leve podem contribuir para os níveis mais elevados da pressão arterial. Após estes resultados, a SAOS foi recentemente incluída como fator causal para a hipertensão arterial sistêmica - HAS em guidelines internacionais. Existem importantes limitações na literatura que não permitem estabelecer uma relação de causalidade entre SAOS e diabetes. Existem evidências demonstrando importante associação da SAOS com acidente vascular cerebral, arritmias e doença cardíaca coronariana, assim como mortalidade cardiovascular ${ }^{6}$.

A Polissonografia - PSG é o melhor método para diagnosticar e avaliar a eficiência dos tratamentos de ronco e apneia. A PSG monitora e quantifica diretamente o número de eventos respiratórios (obstrutivos, centrais ou mistos) e a hipoxemia resultante ${ }^{7}$. A polissonografia padrão utiliza no mínimo sete canais incluindo eletroencefalograma - EEG, eletrooculograma - EOG, eletromiografia EMG submentoniana, eletrocardiograma - ECG, fluxo aéreo oronasal, movimento respiratório e saturação da oxihemoglobina, gravação sonora da medida do ronco, monitorização dos movimentos dos membros através da eletromiografia e vídeo gravado ${ }^{8}$.

Existem relatos de que o diagnóstico por meio da polissonografia tem mostrado episódios de bruxismo frequentemente associados à microdespertares, 
caracterizados por ativação cortical transitória, com aumento simultâneo nas atividades cardíaca e muscular, com duração de 3 a 15 segundos. Os autores sugerem que a origem central dessa parassonia, em geral, a arquitetura do sono no bruxista, é normal, assim como a densidade de sono de ondas lentas. Entretanto os despertares ou microdespertares estão associados aos eventos. A ativação cerebral e cardíaca pouco antes dos eventos de bruxismo também pode ser uma resposta a um quadro respiratório na forma de evento obstrutivo ou restrição do fluxo aéreo?.

A administração da pressão positiva contínua da via aérea - CPAP constitui-se atualmente no padrão-ouro para o tratamento da $\mathrm{SAOS}^{10}$. O CPAP melhora a qualidade do sono, reduz a sonolência diurna excessiva e as alterações cognitivas associadas à $\mathrm{SAOS}^{10,11}$. Apesar de não ser tão efetivo no controle da SAOS quanto o CPAP, os pacientes parecem apresentar melhor adesão aos $\mathrm{AIOs}^{12}$.

A praticidade e o relativo conforto fazem com que os Aparelhos Intraorais (AIO) sejam mais aceitos pelos pacientes em tratamento, sendo indicados para ronco e SAOS de leve a moderada. Mas podem ser uma alternativa para os pacientes com SAOS grave que não se adaptam ao $\mathrm{CPAP}^{13}$.

Os AIO são classificados como: 1) Aparelhos Retentores de Língua - ARL; e 2) Aparelhos de Avanço Mandibular - AAM, dispositivos mais utilizados e investigados na literatura médica e odontológica ${ }^{14}$.

O Aparelho de Controle Lingual é baseado no conceito de controle de posicionamento da língua e é indicado para pacientes com quantidade de dentes insuficientes para ancoragem (e retenção) do dispositivo, para pacientes edêntulos portadores de prótese total, pacientes com reabilitações protéticas extensas, problemas periodontais, presença de disfunção temporomandibular e/ou bruxismo ${ }^{15}$.

De acordo com trabalhos encontrados na literatura, o controle da SAOS por meio do uso de aparelhos intraorais tem-se demonstrado eficaz com indicação secundária também em pacientes com SAOS grave ${ }^{16}$. Henke em 2000, avaliou 28 pacientes portadores de SAOS severa em uso de AIO cujo resultado apresentou uma significativa melhora de $80 \%$ no índice de apneia/hipopneia, salientando inclusive que o índice de apneia/hipopneia severo não deve servir de critério para excluir o paciente do uso de aparelhos intraorais ${ }^{17}$.

Esta pesquisa trata-se de um estudo descritivo tipo relato de caso, observacional, aprovado pelo comitê de ética em pesquisa da Universidade Federal de Pernambuco - UFPE.

Participou do estudo um paciente do gênero masculino, de 65 anos, hipertenso, que procurou a Clínica de Extensão de Tratamento Multidisciplinar de Tratamento de Bruxismo/ Apneia da Odontologia da UFPE, após encaminhamento médico para tratamento de ronco e apneia do sono grave.

De acordo com a primeira PSG realizada, o IMC era de $25 \mathrm{Kg} / \mathrm{m}^{2}$, parâmetros respiratórios compatíveis com: IAH de 32,23 ev/h, presença de ronco moderado, dessaturação média de $96 \%$ e mínima de $79 \%$, distribuição do sono equivalente a $\mathrm{N} 1=4,5 \%, \mathrm{~N} 2=77,4 \%, \mathrm{~N} 3=6,5 \%$ (redução), REM $=11,5 \%$ (redução), microdespertares de 224, Latência de REM de 131,0 e TTS (Tempo Total de Sono) de 7,38h.

O paciente relatava sono inadequado, cansaço diurno, e não ter se adaptado ao tratamento com o CPAP; apresentavase hiperativo e ansioso e, ao exame clínico intraoral, constatou-se presença de várias coroas cerâmicas unitárias na arcada superior e palato ogival.

A confecção do AIO de controle lingual seguiu as seguintes etapas: moldagem da arcada superior com alginato (Hidrogum, Zhermack; Rovigo, Itália), desinfecção do molde em solução de hipoclorito de sódio a $1 \%$ durante 10 minutos, lavagem e secagem do molde e obtenção do modelo em gesso pedra tipo IV (Durone - Dentsply - São Paulo Brasil), sob vibração para minimizar a formação de bolhas, recorte e preparação do modelo (eliminação das retenções), plastificação (placa de acetato cristal $2 \mathrm{~mm}$, Bio-Art), recorte da placa, ajuste na boca, toques bilaterais contra os dentes antagonistas), seleção da mola (redonda), fixação da mola na placa (fio ortodôntico de aço inox $2 \mathrm{~mm}$, Moreli), ajuste inicial da mola na boca (deglutição permitida) e inserção do fio de nylon $1 \mathrm{~mm}$ ligando o acrílico da haste ao corpo da placa como medida de segurança.

O acessório lingual, agente responsável pelo controle direto do posicionamento da lingua, é composto por um fio calibre 1,0 ou 1,2 mm, dobrado em forma de "S" duplo e incorporado à placa superior. Em sua extremidade inferior é incorporada uma superfície de resina acrílica, de vários possíveis formatos, e que se posiciona na base da língua. $\mathrm{O}$ acessório tem por finalidade não só escorar a língua, mas também promover sua anteriorização de modo a impedir seu retroposicionamento e garantir a patência da via aérea orofaringeana. A pressão e o posicionamento da mola devem ser minuciosamente calibrados em sessões semanais, caso contrário o desconforto provocado resultará em inadaptação. A pressão sobre a língua deve ser efetivada por meio de ativações no "S", com intuito de alongar o fio em cerca de $1 \mathrm{~mm}$. O posicionamento do acessório deverá ser também semanalmente retificado em direção posterior, até o limite de aceitação do paciente e esta evolução da mola em direção à orofaringe foi acompanhada com teradiografia. Nos casos mais complexos esse procedimento poderá se repetir por até seis meses. 
Os pacientes com maior tolerância ao reflexo de vômito têm um excelente prognóstico, haja vista a grande amplitude de ajuste que permite esse mecanismo (Figuras 1 a 4$)$.

Figura 1: Componentes do AIO de Controle Lingual: a) superfície de resina acrílica (mola), b) fio inox, c) placa superior e d) fio de segurança

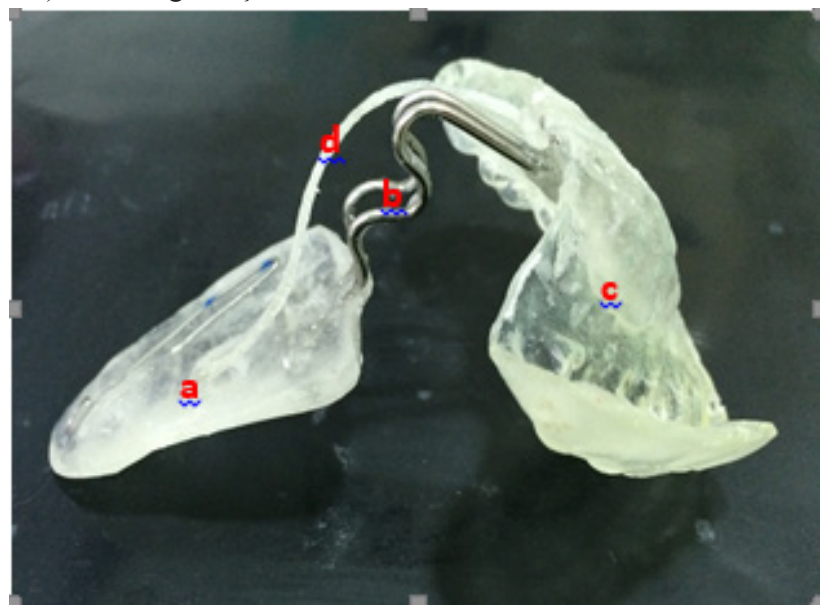

Fonte: Os autores.

Figura 2: AIO de Controle Lingual (vista lateral)

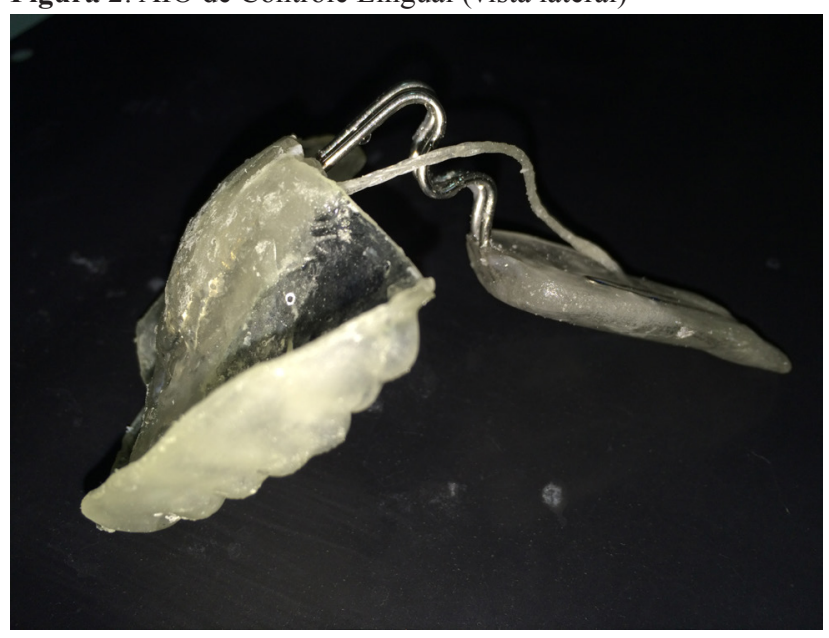

Figura 3: AIO de Controle Lingual Inserido na Boca

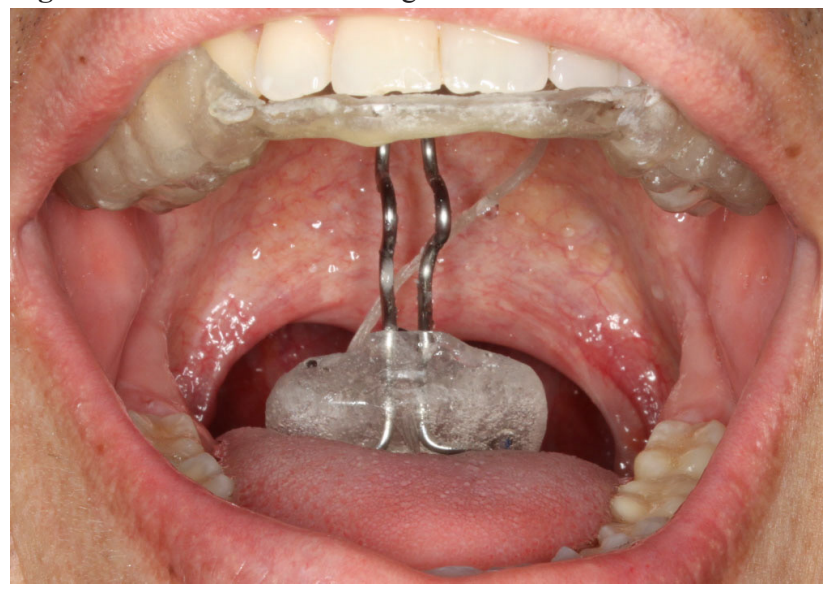

Fonte: Os autores.
Figura 4: Telerradiografia de controle da posição da mola

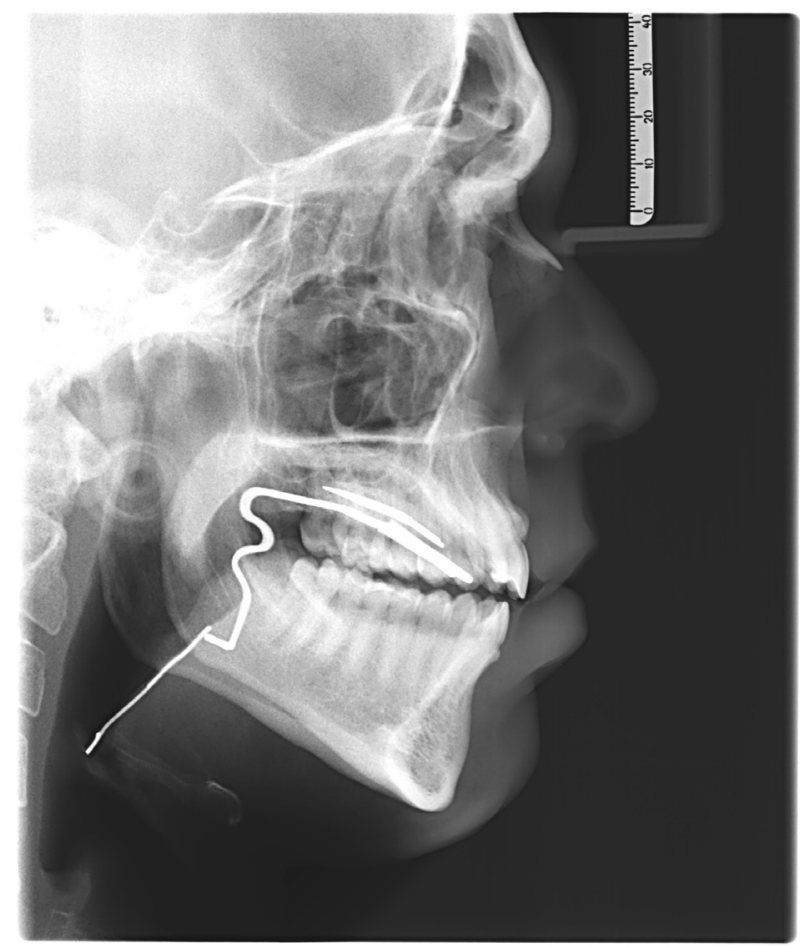

Fonte: Os autores.

O paciente relatou aumento da salivação no início do uso e dificuldade para deglutição no período de adaptação.

Para o exame da movimentação mandibular e complicações funcionais estomatognáticas, lançou-se mão da eletrognatografia - EGN realizada no Laboratório de Motricidade da Fonoaudiologia da UFPE. A EGN é um exame tridimensional computadorizado, objetivo e não invasivo, capaz de avaliar milimetricamente todos os movimentos mandibulares e suas respectivas funções e pode servir como auxiliar no diagnóstico de anormalidades nas funções da ATM. O equipamento utilizado foi eletrognatógrafo $J T-3 D$ da BioRESEARSH® e o software empregado na leitura dos dados captados na EGN é o BioPAKSistem da SQUIB do Brasil S/A, que estará disponível em um notebook da marca $L G$ com sistema operacional Windows Vista Home Premium ${ }^{\circledR}$, HD de $110 \mathrm{~GB}$, processador Intel Dual-Core Inside ${ }^{\circledR}$ 1,6 GHz, memória RAM de 2 GB, 32 BITs. Um cabo USB será usado para conexão do eletrognatógrafo com o notebook.

O eletrognatógrafo é composto de sensores fixos ao crânio do paciente, capazes de detectar a presença de um campo magnético cujo imã é posicionado sobre as faces vestibulares dos incisivos centrais inferiores sem interferir na oclusão. Os traçados fornecidos pelo estudo deverão proporcionar ao clínico um auxílio diagnóstico e uma melhor visualização da movimentação mandibular nos planos horizontal, sagital e frontal.

A análise polissonográfica e eletrognatográfica foram realizadas antes do início do tratamento e após cinco meses de tratamento com o AIO (Figuras 5 a 8). 
Figura 5: Exame Eletrognográfico depois do uso do AIO: vista sagital, amplitude de movimento mandibular

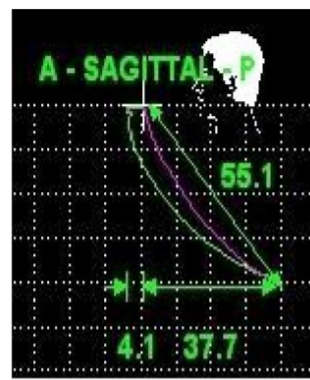

PRÉ

\begin{tabular}{|c|c|c|c|c|}
\hline \multicolumn{5}{|c|}{ MÁXIMA ABERTURA MANDIBULAR PRÉ } \\
\hline $\begin{array}{c}\text { Máximo } \\
\text { Deslocamento } \\
\text { Atingido }\end{array}$ & Vertical & Oblíquo & Propulsão & Retrusão \\
\cline { 2 - 5 } & $40 \mathrm{~mm}$ & $55.1 \mathrm{~mm}$ & $4.1 \mathrm{~mm}$ & $37,7 \mathrm{~mm}-$ \\
\hline \multicolumn{5}{|c|}{ MÁxIMA ABERTURA MANDIBULAR Pós } \\
\hline $\begin{array}{c}\text { Máximo } \\
\begin{array}{c}\text { Deslocamento } \\
\text { Atingido }\end{array}\end{array}$ & Vertical & 0blíquo & Propulsão & Retrusão \\
\cline { 2 - 5 } & $\mathbf{4 7 . 2 \mathrm { mm }}$ & $57.1 \mathrm{~mm}$ & $2.0 \mathrm{~mm}$ & $31,2 \mathrm{~mm}=$ \\
\hline
\end{tabular}

Fonte: Dados da pesquisa.

Figura 6: Exame Eletrognográfico antes do uso do AIO: vista sagital, amplitude de movimento mandibular



PRÉ

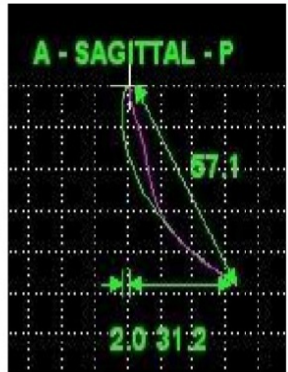

Pós

\begin{tabular}{|c|c|c|c|c|}
\hline \multicolumn{5}{|c|}{ MÁXIMA ABERTURA MANDIBULAR PRÉ } \\
\hline $\begin{array}{c}\text { Máximo } \\
\text { Deslocamento } \\
\text { Atingido }\end{array}$ & Vertical & Obliquo & Propulsão & Retrusão \\
\cline { 2 - 5 } & $40 \mathrm{~mm}$ & $55.1 \mathrm{~mm}$ & $4.1 \mathrm{~mm}$ & $37,7 \mathrm{~mm}-$ \\
\hline \multicolumn{5}{|c|}{ MÁXIMA ABERTURA MANDIBULAR Pós } \\
\hline $\begin{array}{c}\text { Máximo } \\
\text { Deslocamento } \\
\text { Atingido }\end{array}$ & Vertical & Obliquo & Propulsão & Retrusão \\
\cline { 2 - 5 } & $47.2 \mathrm{~mm}$ & $57.1 \mathrm{~mm}$ & $2.0 \mathrm{~mm}$ & $31,2 \mathrm{~mm}-$ \\
\hline
\end{tabular}

Fonte: Dados da pesquisa.

Figura 7: Exame eletrognográfico antes do uso do AIO



Fontes: Dados da pesquisa.
Figura 8: Exame eletrognográfico após o uso do AIO

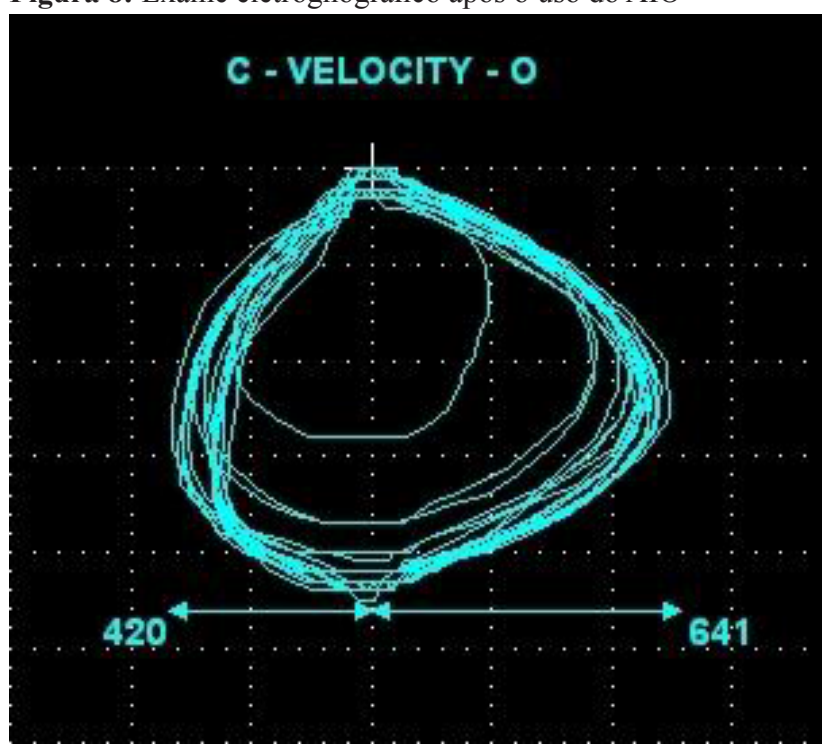

Fontes: Dados da pesquisa.

\section{Resultados e Discussão}

De acordo com trabalhos encontrados na literatura, o controle da SAOS por meio do uso de aparelhos intraorais tem-se demonstrado eficaz com indicação secundária também em pacientes com SAOS grave ${ }^{16}$. Henke, em 2000, avaliou 28 pacientes portadores de SAOS severa em uso de AIO cujo resultado apresentou uma significativa melhora de $80 \%$ no índice de apneia/hipopneia salientando, inclusive, que o índice de apneia/hipopneia severo não deve servir de critério para excluir o paciente do uso de aparelhos intraorais ${ }^{17}$.

O sono é um componente básico e essencial da regulação biológica e homeostática do organismo. No decorrer de uma noite de sono, os sistemas e as funções fisiológicas sofrem alterações acompanhando os ciclos ultradianos. A cada momento do sono (NREM e REM), as respostas do organismo são diferentes, o que permite compreender os distúrbios do sono. O sono de ondas lentas (N3) é extremamente repousante e está associado à redução dos tônus vasculares periféricos. Neste estágio do sono ocorre uma redução significativa da pressão arterial sistêmica. Durante o sono REM, observamse intensas flutuações tanto da pressão arterial sistêmica quanto da frequência cardíaca, que de tempos em tempos sofrem elevações bruscas. Durante esses episódios ocorrem descargas adrenérgicas intensas que resultam em elevação da pressão arterial sistêmica e frequência cardíaca ${ }^{18}$. A descrição do nosso caso revelou um paciente hipertenso e ansioso, provavelmente em decorrência do descontrole do sono de ondas lentas e latência de REM, embora o tempo total do sono tenha se revelado dentro da normalidade. Após cinco meses de uso do AIO o paciente revelou que sua pressão está controlada em 15/8 mm de Hg, que faz uso da medicação Micardis/Anlo 80/5, mudou hábitos de alimentação e passou a se exercitar, pelo menos, por 2 horas/dia.

As revisões sistemáticas Cochrane publicadas em 2011 
por Ahrens et al. demonstram haver evidências científicas crescentes, sugerindo que o tratamento com AIO melhora os sintomas subjetivos de sonolência e reduz significantemente os eventos de apneia e saturação mínima de oxihemoglobina quando comparados com aparelhos placebo. O uso desses aparelhos pode, em alguns pacientes, eliminar e/ou reduzir o ronco e aumentar o volume das vias aéreas respiratórias ${ }^{19}$. Neste trabalho, durante o acompanhamento do paciente por cinco meses, o seu IAH passou de $32,23 \mathrm{ev} / \mathrm{h}$ para $11,73 \mathrm{ev} / \mathrm{h}$, de 224 microdespertares para 19 microdespertares, o ronco de moderado a leve, o que significa que o seu quadro de apneia, que era considerado severo, regrediu para uma apneia de grau leve. Houve recuperação da fase do Sono de Ondas Lentas (N3) e da Latêncis de sono REM (Quadro 1).

Quadro 1: Resultados polissonográficos do tratamento com AIO

\begin{tabular}{|l|l|}
\hline Basal & 5 Meses Depois \\
\hline $\mathrm{IMC}=25 \mathrm{~kg} / \mathrm{m}^{2}$ & $\mathrm{IMC}=26,23 \mathrm{~kg} / \mathrm{m}^{2}$ \\
\hline Idade $=65$ & Idade $=65$ \\
\hline $\mathrm{IAH}=32,23 \mathrm{ev} / \mathrm{h}$ & $\mathrm{IAH}=11,73 \mathrm{ev} / \mathrm{h}$ \\
$\mathrm{N} 1=4,5 \%$ & $\mathrm{~N} 1=5,5 \%$ \\
$\mathrm{~N} 2=77,4 \%$ & $\mathrm{~N} 2=60,9 \%$ \\
$\mathrm{~N} 3=6,5 \%($ redução) & $\mathrm{N} 3=23,1 \%$ \\
$\mathrm{REM}=11,5 \%($ redução) & $\mathrm{REM}=10,5 \%($ redução $)$ \\
\hline $\mathrm{SaO} 2=96 \%$ & $\mathrm{SaO} 2=97 \%$ \\
\hline $\mathrm{SaO} 2$ min $=79 \%$ & $\mathrm{SaO} 2$ min $=80 \%$ \\
\hline $\mathrm{Ronco}$ moderado & $\mathrm{Ronco}$ leve \\
\hline $\mathrm{Microdespertares}=224$ & Microdespertares $=19$ \\
Latência de REM $=131,0$ & Latência de REM $=70,5$ \\
TTS $=7,38 \mathrm{~h}$ & TTS $=7,0 \mathrm{~h}$ \\
\hline
\end{tabular}

Fonte: Dados da pesquisa.

O AIO de controle lingual, por prescindir do avanço mandibular e atuar diretamente sobre a língua, permite que esse mecanismo possa ser utilizado por pacientes com dor temporomandibular - DTM, portadores de prótese total, portadores de próteses parciais removíveis, próteses extensas, periodontopatias e poucos elementos dentários nas arcadas sem ocasionar problemas de adaptação. O AIO de controle lingual também tem indicação para aqueles pacientes que sofrem de maior desconforto muscular devido à presença de bruxismo ${ }^{15}$, que frequentemente apresentam número de movimentação mandibular três vezes maior quando comparados aos não bruxistas. Alguns pacientes apresentam intolerância à mudança de postura ortopédica ${ }^{16}$, podendo assim serem beneficiados com o AIO de controle lingual por não necessitar da ancoragem mandibular. Este aparelho foi idealizado pelo Doutor Jorge Machado Caram sob a patente de número PI 0101407-2, ortodontista, aprovado na primeira Prova de Certificação em Odontologia do Sono da ABS (Associação Brasileira do Sono), professor convidado dos Cursos de Capacitação Odontológica em Medicina do Sono no Centro de Pesquisas Odontológicas, São Leopoldo Mandic - São Paulo/SP.

Foi demonstrado que alguns aspectos que interferem na efetividade dos aparelhos no tratamento com AIO referem-se ao modelo selecionado, ao material de confecção, ao método de fabricação e ao que permite liberdade de movimentação mandibular ${ }^{19}$. A mola lingual do aparelho tem por finalidade "amparar" a língua durante sua "queda" ao adormecer, garantindo sua anteriorização em meio a sua hipotonia muscular típica dos estágios profundos do sono, aumentando assim a permeabilidade da VAS. A pressão e o posicionamento da mola lingual devem ser minuciosamente calibrados em sessões semanais, até o limite de aceitação do paciente ou o mais próximo possível da base da língua ${ }^{15}$.

Estudos mostraram que os efeitos sobre a saúde relacionados aos aspectos de qualidade de vida e desempenho cognitivo foram semelhantes entre $\mathrm{AIO}$ e $\mathrm{CPAP}^{12}$, o que ficou retratado em nosso trabalho quando o paciente ao substituir o CPAP pelo AIO de controle Lingual foi acometido de diminuição dos microdespertares de 224 para 19 permitindo, segundo relato do paciente, melhor mobilidade física, desenvoltura social e emocional.

Os efeitos colaterais dos AIOs normalmente são leves e transitórios ${ }^{21,23}$. Recente estudo randomizado avaliou intensidade da dor e prejuízos na função mandibular com AIO e CPAP após dois meses, um ano e dois anos. Foi observado que o tratamento com AIO resultou em mais dor por DTM que o CPAP no período inicial de uso, muito embora a dor tenha natureza transitória e, em geral, não seja grave. Não ocorreram, porém, limitações na função mandibular durante os dois anos com ambos os tratamentos ${ }^{21}$. A EGN é um exame tridimensional computadorizado, objetivo e não invasivo capaz de avaliar milimetricamente todos os movimentos mandibulares e suas respectivas funções e pode servir como auxiliar no diagnóstico de anormalidades nas funções da ATM. Em nosso trabalho não foi registrado nenhum efeito limitante ou diferenças significativas na máxima abertura vertical, propulsão e retrusão mandibular comprovados pela eletrognatografia quando comparados antes e após cinco meses de uso do AIO de controle lingual. O aumento na amplitude vertical do movimento é interpretado como positivo, uma vez que possa ter ocorrido uma maior harmonia e liberação entre o disco e a eminência articular no movimento de abertura. A diminuição da propulsão e da retrusão da mandíbula nos respectivos momentos de abertura e fechamento podem ser interpretados como benéficos à diminuição da SAOS, já que a mandíbula se distancia, talvez por adaptação, das estruturas da orofaringe nesses movimentos (Figuras 5 e 6 e Quadro 2).

Quadro 2: Valores da eletrognatografia antes e após o uso do AIO na abertura mandibular (plano sagital)

\begin{tabular}{|c|c|c|c|c|}
\hline \multicolumn{5}{|c|}{ Máxima Abertura Mandibular Pré } \\
\hline $\begin{array}{c}\text { Máximo } \\
\text { Deslocamento } \\
\text { Atingido }\end{array}$ & Vertical & Oblíquo & Propulsão & Retrusão \\
\cline { 2 - 5 } & $35,6 \mathrm{~mm}$ & $55,1 \mathrm{~mm}$ & $4,1 \mathrm{~mm}$ & $37,7 \mathrm{~mm}$ \\
\hline \multicolumn{5}{|c|}{ Máxima Abertura Mandibular Pós } \\
\hline $\begin{array}{c}\text { Máximo } \\
\text { Deslocamento } \\
\text { Atingido }\end{array}$ & Vertical & Oblíquo & Propulsão & Retrusão \\
\cline { 2 - 5 } & $47,2 \mathrm{~mm}$ & $57,1 \mathrm{~mm}$ & $2 \mathrm{~mm}$ & $32,2 \mathrm{~mm}$ \\
\hline
\end{tabular}

Fonte: Dados da pesquisa. 
Com respeito à avaliação milimétrica do ciclo mastigatório, no mesmo estudo não foram notadas diferenças significativas antes e após o tratamento. Apenas a velocidade de abertura de boca foi reduzida e a amplitude oblíqua de abertura de boca se tornou mais homogênea. Vale chamar atenção para o balanceio entre os lados de trabalho e o de não trabalho durante a mastigação pós-tratamento (Figuras 7 e 8 e Quadro 3).

Quadro 3: Valores da eletrognatografia antes e após o uso do AIO na máxima velocidade de abertura e fechamento mandibular atingida

\begin{tabular}{|c|c|c|}
\hline \multicolumn{3}{|c|}{ Máxima Abertura Mandibular Pré } \\
\hline Movimento & Fechamento & Abertura \\
\hline Velocidade em mm/s & $449 \mathrm{~mm} / \mathrm{s}$ & $661 \mathrm{~mm} / \mathrm{s}$ \\
\hline \multicolumn{2}{|c|}{ Máxima Abertura Mandibular Pós } \\
\hline Movimento & Fechamento & Abertura \\
\hline Velocidade em mm/s & $420 \mathrm{~mm} / \mathrm{s}$ & $641 \mathrm{~mm} / \mathrm{s}$ \\
\hline
\end{tabular}

Fonte: Dados da pesquisa.

Sugere-se avaliação gradual para melhor comparação do movimento mandibular e da mastigação. O aparelho, no geral, é bem tolerado e pode promover redução do IAH confirmado pelo exame polissonográfico antes e após o uso dele ${ }^{12}$.

\section{Conclusão}

Conclui-se que, nas condições deste trabalho de relato de caso, durante o acompanhamento do paciente por cinco meses, o quadro de apneia severa regrediu para uma apneia de grau leve, o ronco passou de moderado a leve, o microdespertar diminuiu de 224 para 19; sugerindo que o dispositivo intraoral de controle lingual pode ser uma alternativa recomendada para o tratamento da SAOS. A continuação da investigação com aumento e padronização da amostra se faz necessária para confirmação dos dados aqui relatados. Essa terapia necessita, além do controle polissonográfico, de revisões e acompanhamento sistemático, tanto do médico, fonoaudiólogo quanto do cirurgião-dentista.

\section{Referências}

1. American Sleep Disorders Association Standards of Practice Committee. Practice parameters for the treatment of snoring and obstructive sleep apnea with oral appliances: An update for 2005. Sleep 2006;29(2):240-3.

2. Martins AB, Tufik S, Moura SMGPT. Physiopathology of obstructive sleep apnea-hypopnea syndrome. J Bras Pneumol 2007;33(1):93-100.

3. Epstein LJ, Kristo D, Strollo PJ Jn, Friedman N, Malhotra A, Patil SP, et al. Clinical guideline for the evaluation, management and long-term care of obstructive sleep apnea in adults. J Clin Sleep Med 2009;5(3):263-76.

4. Lévy P, Tamisier R, Minville C, Launois S, Pépin JL. Sleep Apnea syndrome in 2011: current concepts and future directions. Eur Respir Rev 2011;20(121):134-46.

5. Martinho FL, Tangerina RP, Moura SM, Gregório LC, Tufik S, Bittencourt LR. Systematic head and neck physical examination as a predictor of obstructive sleep apnea in classe III obese patients. Braz J Med Biol Res 2008;41:1093-7.
6. Kendzerska T, Mollayeva T, Gershon AS, Leung RS, Hawker G, Tomlinson G. Untreated obstructive sleep apnea and the risk for serious long-term diverse outcomes a systematic review. Sleep Med Rev 2014;8(1):49-59. doi: 10.1016/j. smrv.2013.01.003

7. Godolfim LR. Apneia obstrutiva do sono. J Bras Ortodon Ortop Facial 2006;11(65/66)484-501.

8. Dal-Fabbro C. Avaliação clínica e polissonográfica do aparelho BRD no tratamento da Síndrome da Apneia Obstrutiva do Sono. Dental Press J Orthod 2010;15(1):75-81.

9. Dal-Fabbro C, de Siqueira JT, Tufick S, Long-term PSG in a bruxism patient: the role of daily anxiety. Slleo Med 2009;10(7):813.

10. Silva RZM, Duarte RLM, Silveira FJM. Tratamento da apneia obstrutiva do sono com pressão positiva contínua na via aérea. Pulmão RJ 2010;19(3/4):83-7.

11. Loredo JS, Ancoli-Israel S, Kim EJ, Lim WJ, Dimsdale JE. Effect of continuous positive airway pressure versus supplemental oxygen on sleep quality in obstructive sleep apnea: a placebo - CPAP- controled study. Sleep 2006;29(4):564-71.

12. LI W, Xiao L, Hu J. The comparision of CPAP and oral appliance in treatment of patients with OSA a systematic review and meta-analysis. Respir Care 2013;58(7):184-95.

13. Carneiro VSM, Alves J, Catão MHCV, Ribeiro RA, Silva RS. Perfil do Paciente Usuário de Aparelho de Protrusão Mandibular. Rev Bras Cienc Saúde 2011;15(2):167-76.

14. Krishnan VMD, Collop AN, Scherr SC. An evaluation of a titration strategy for prescription of oral appliances for obstructive sleep apnea. Chest 2008;133(5):1135-41.

15. Caram JM, Quintela MM. A órtese lingual - uma nova proposta de aparelho intraoral para apneia do sono grave: relato de casos. Orthod Sci Orthod Sci Pract 2013;6(21):1107.

16. Dal-Fabbro C, Chaves JCM, Tufick S. A odontologia na medicina do sono. São Paulo: Dental Press; 2012.

17. Henke KG, Frantz DE, Kuna ST. An oral elastic mandibular advancement device for obstructive sleep apnea. Am J Respir Crit Care Med 2000;161(2):420-5.

18. Andersen ML, Bittencourt LRA. Fisiologia do sono. Tufik S. Medicina e biologia do sono. São Paulo: Manole; 2008. p.4858.

19. Ahrens A, McGrath C, Hagg U. A systematic review of the efficacy of oral appliance design in the management of obstructive sleep apnea. Eur J Orthod 2011;33(3):318

20. Giles TL, Lasserson TJ, Smith BH, White J, Wright J, Cates CJ. Continuous positive airways pressure for obstructive sleep apnea in adults. Cochrane Database Syst Rev 2006,19;(3):CD001106.

21. Doff MH, Veldhuis SK, Hoekema A. Long-term oral appliance therapy in obstructive sleep apnea syndrome: a controlled study on temporomandibular side effects. Clin Oral Investig 2012;16(3):689-97.

22. Luz, JM. Medição de deslocamento através de sensores magnetoresistivos aplicada ao movimento mandibular. Tese [Doutorado em Engenharia] - Universidade Federal do Rio Grande do Sul; 2002. 